\title{
Abstracts of scientific presentations at the 2016 Annual National Conference of the Critical Care Society of Southern Africa
}

\section{Does prone positioning recruit dorsal lung regions in children with acute respiratory distress syndrome (ARDS)?}

\author{
$\underline{\text { A Lupton-Smith }},{ }^{1 \star}$ A Argent, ${ }^{1}$ P Rimensberger, ${ }^{2}$ B Morrow ${ }^{1}$ \\ ${ }^{1}$ School of Child and Adolescent Health, Faculty of Health Sciences, \\ University of Cape Town, South Africa \\ ${ }^{2}$ Paediatric and Neonatal Intensive Care Unit, University Hospital of \\ Geneva, Switzerland \\ *aluptonsmith@gmail.com
}

Background. Prone positioning is commonly used in patients with acute lung injury (ALI)/ARDS to improve oxygenation. Improved oxygenation is thought to occur due to recruitment of collapsed dorsal lung regions and improved ventilation homogeneity.

Objective. To determine the effects of prone turning on regional ventilation distribution and homogeneity in children with ALI/ARDS Methods. Thoracic electrical impedance tomography (EIT) measurements were taken in the supine position (baseline) and 5, 20 and 60 minutes after being turned into the prone position. Arterial blood gas measurements were obtained at baseline and after 60 minutes of being in the prone position. Repeated measures analysis of variance (ANOVA) was used to determine the difference in mean relative impedance change and ventilation homogeneity between responders and non-responders.

Results. Fourteen participants $(n=9,64 \%$ male) with a median (interquartile range) age of 20 (11.6 - 26.2) months were studied. Seven $(50 \%)$ participants showed an improved $\mathrm{PaO}_{2}$ of $2.1(1.5-3.3)$ $\mathrm{kPa}$ ('positive responders'), while seven showed a reduction of 1.1 (0.5-1.8) $\mathrm{kPa}$ ('negative responders') after 60 minutes. Proportion of ventilation in the dorsal lung was no different between responders and non-responders $(p=0.59)$. Responders showed significantly more variability in ventilation inhomogeneity at baseline compared with non-responders $(p=0.005)$. After 60 minutes, ventilation inhomogeneity was significantly less variable in the responders $(p=0.02)$.

Conclusion. Prone positioning did not result in recruitment of the dorsal lung regions, but rather more homogenous ventilation. Children who responded to prone positioning had more variable ventilation inhomogeneity at baseline, compared with those who did not respond.

\section{Prognosis of myocardial infarction and myocardial injury following vascular surgery}

\author{
T Kisten, $_{2}{ }^{\star}$ B M Biccard ${ }^{2}$ \\ ${ }^{1}$ Discipline of Anaesthesia and Critical Care, School of Clinical \\ Medicine, Nelson R Mandela Medical School, University of \\ KwaZulu-Natal, Durban, South Africa \\ ${ }^{2}$ Department of Anaesthesia and Perioperative Medicine, Faculty of \\ Health Sciences, University of Cape Town, South Africa \\ *toshiekis@gmail.com
}

Background. Perioperative myocardial infarction (PMI) is the most common major cardiovascular complication in non-cardiac surgery, and increases perioperative mortality. Prognostically relevant myocardial injury following non-cardiac surgery (MINS) was shown to be an independent predictor of 30-day mortality. The VISION, POISE and MINS studies have highlighted the need for routine troponin surveillance in high-risk non-cardiac surgery. Studies in vascular patients show an increased incidence and mortality from perioperative myocardial infarction.

Objectives. The primary objective was the incidence of PMI and MINS in patients admitted to intensive care following vascular surgery, and the in-hospital mortality in those who experienced a PMI or MINS. The secondary objective was to identify perioperative predictors of PMI or MINS.

Methods. A retrospective review was conducted of all patients aged at least 45 years between 1 January 2011 and 31 December 2013 admitted to Inkosi Albert Luthuli Central Hospital intensive care unit $2 \mathrm{~B}$ following vascular surgery. A total of 140 patients were included in the final analysis. Demographics, cardiovascular risk factors, troponin I levels for 3 days following surgery, perioperative brain natriuretic peptide (BNP), electrocardiogram and echocardiogram findings were extracted. The third universal definition was used for diagnosis of myocardial infarction. Patients who had a troponin level above normal but below the diagnostic threshold for a myocardial infarction were classified as MINS. Alternative or non-ischaemic causes of a troponin elevation below the myocardial infarction diagnostic threshold were not classified as MINS.

Results. A total of $24.3 \%$ of the patients had a PMI and a further $25 \%$ had MINS. Compared with the in-hospital mortality of $18.3 \%$ in patients without troponin elevation, PMI was associated with significantly increased in-hospital mortality of $58.8 \%(p<0.01)$ while MINS was not $(17.1 \%, p=1.00)$. Increasing age, the highest postoperative BNP and a blood transfusion within the first 3 days postoperatively were independent predictors of PMI. PMI and a history of congestive cardiac failure were independent predictors of in-hospital mortality. Conclusion. Perioperative myocardial infarction and MINS were present in nearly half the study population. A vascular surgical patient with a PMI, but not MINS, had significantly increased in-hospital mortality.

\section{Postoperative outcome of intensive care unit (ICU) surgical patients at a Zimbabwean hospital}

\author{
C Tadyanemhandu, ${ }^{1 *}$ D Shoko, ${ }^{1}$ C Nhunzvi, ${ }^{1}$ S Chengetanai, ${ }^{2}$ \\ V Chikwasha \\ ${ }^{1}$ Department of Rehabilitation, College of Health Sciences, University \\ of Zimbabwe, Harare \\ ${ }^{2}$ Division of Basic Medical Sciences, Faculty of Medicine, National \\ University of Science and Technology, Bulawayo, Zimbabwe \\ *cathytadya@gmail.com
}


Background. About $10-15 \%$ of hospital admissions in developing countries are due to diseases amenable to surgical treatment. It is estimated that surgical conditions account for $11 \%$ of the total global burden of disease and 25 million disability-adjusted life years (DALYs) in Africa, the region with the highest concentration of surgical DALYs (38/1 000 population). HIV infection has increased the burden of surgical practice by overloading the practice and increasing related surgical diseases, which have resulted in more challenges being faced in surgical interventions of HIV-infected patients and methods used to improve their outcomes.

Objectives. To describe the demographic characteristics of postsurgical patients admitted to the ICU, the associated comorbidities in the patients and the outcomes.

Methods. A retrospective review of records of patients who were admitted into Parirenyatwa ICU from the operating room between 1 January 2015 and 30 September 2015 was done. The following data were collected: demographic data, surgery history, comorbidities and complications that developed.

Results. Out of the 119 patients' records accessed, the majority ( $n=62$, $52.1 \%$ ) were males and the mean age of patients was 42.8 years (standard deviation (SD) 18.3). The reason for ICU admission was mainly for cardiopulmonary support using invasive and non-invasive ventilation $(n=71,59.7 \%)$. The majority of the patients were from general surgery ( $n=52,43.7 \%)$, followed by neurosurgery patients $(n=32,26.9 \%)$. The most performed surgery was laparotomy, in 62 of the patients (52.1\%), followed by craniotomy, in 29 of the patients $(24.4 \%)$. The majority were elective surgeries $(n=70,58.8 \%)$, with the reasons for surgery tumours $(n=49,41.1 \%)$, infection $(n=29,24.4 \%)$ and trauma $(n=19$, $16.0 \%)$. Respiratory complications were noted in the majority of the patients $(n=61,51.3 \%)$ post surgery. Fourteen $(11.8 \%)$ of the patients were HIV-positive. The other common comorbidities were hypertension and diabetes. The mean (SD) length of stay in ICU was 3.8 (3.8) days. The mortality rate was $21.8 \%$. Mortality was associated with HIV-positive status ( $p=0.001)$, emergency surgery $(p=0.001)$, respiratory complications $(p<0.001)$ and ICU admission for cardiopulmonary support $(p<0.001)$.

Conclusion. The postoperative management of surgical patients is further challenged by comorbidities such as hypertension, diabetes and HIV. Strategies to reduce surgical morbidity and mortality have to be formulated to improve outcome of patients.

\section{Yellow fever outbreak: Experience in an Angolan intensive care unit (ICU)}

\author{
$\underline{\mathrm{N} \text { Velez }}{ }^{\star}$ F Rodriguez, R Alfredo, D Gaspar, E Viegas, E Filipe, \\ E Tomás \\ Clínica Sagrada Esperança - Ilha do Cabo, Luanda, Angola \\ *velez.nadia@gmail.com
}

Background. On 21 January 2016, Angola notified the World Health Organization of a yellow fever outbreak. The first case, with onset date 5 December 2015, was identified in Viana municipality. By 17 April 2016, 1708 suspected cases, including 238 deaths (case fatality rate $13.9 \%)$, had been reported from 16 provinces.

Objectives. To determine clinical characteristics and outcomes of yellow fever among critically ill patients admitted to Clínica Sagrada Esperança's intensive care unit.

Methods. A retrospective review of patient records was conducted. Patients admitted with laboratory confirmation of yellow fever were included in the study. The diagnostic tests performed were
IgM antibody capture enzyme-linked immunosorbent assay (MACELISA) and polymerase chain reaction (PCR). The demographic and clinical characteristics, laboratory parameters and survival were analysed.

Results. From the 39 suspected cases admitted, 16 had laboratory confirmation of yellow fever. Eleven were adults and five were infants, mainly males (68\%). On admission, mean (standard deviation) sequential organ failure assessment (SOFA) was 9.56 (2.70), and simplified acute physiology score (SAPS) 3 was 63.87 (7.17), with a mean predictive death rate of $44 \%$. On examination, all the patients presented with fever, shivers, headaches, weakness, nausea, vomiting, jaundice and consciousness disorders. Eight (56.2\%) had haemorrhagic manifestations. Half of them were coinfected with Plasmodium falciparum malaria, 4 (25\%) with dengue fever, 1 with leptospirosis and 1 with hepatitis B virus. Mean length of stay was 6 (5.9) (range 1 - 20) days. On the first day, $87.5 \%$ presented with bilirubin above $6 \mathrm{mg} / \mathrm{dL}$ and creatinine above $4 \mathrm{mg} / \mathrm{dL}$. During their stay, $93.75 \%$ $(n=15)$ required ventilatory and vasopressor support, and $53.75 \%$ $(n=9)$ required renal support. From the $12(75 \%)$ who died, six deaths occurred in the first 48 hours.

Conclusion. Yellow fever is a hyperacute disease with a very high mortality, even with advanced life support. Therefore, the only way to avoid this fatal outcome is prevention.

\section{Paediatric cardiac critical care admissions to a tertiary paediatric intensive care unit (PICU)}

\section{B Rossouw,${ }^{*}$ M Wege, A C Argent, B Morrow}

Division of Critical Care and Children's Heart Diseases, Red Cross War Memorial Children's Hospital, University of Cape Town, South Africa

*beyra.rossouw@uct.ac.za

Background. Paediatric cardiac critical care (PCCC) in South Africa is not well described.

Objectives. This is the first study describing the patient profiles and treatment of PCCC admissions to a South African tertiary mixed medical and surgical PICU.

Methods. A prospective review was conducted of consecutive patients admitted to the Red Cross War Memorial Children's Hospital PICU with a cardiac discharge diagnosis from January 2015 to December 2015.

Results. A total of 472 PCCC PICU admissions of 409 patients were included. Of these, 274 admissions followed elective cardiac surgery, 37 were post elective general surgery and 161 admissions were unplanned emergency admissions. Indications for emergency admission included: shock (28\%), respiratory support required (22\%), cyanosis (18\%), decompensated cardiac failure (18\%), post cardiac arrest (8\%) and life-threatening arrhythmias (3\%). Comparing elective admissions with emergency admissions: the median age of admission was $24 \mathrm{v}$. 5 months $(p<0.0001)$; length of PICU stay 3 v. 6 days $(p<0.0001)$; length of ventilation $1 \mathrm{v} .2$ days ( $p=0.13$ ), length of inotrope use $1 \mathrm{v}$. 2 days ( $p=0.08)$; and median maximum inotrope score $1 \mathrm{v} .9(p=0.46)$. A cardiac diagnosis was made for the first time in the PICU in 57 patients. A total of 85 emergency theatre procedures (32 cardiac surgical) were undertaken from the PICU. Eighty-one patients needed PICU readmissions and 19 needed redo cardiac surgery during this study period. PICU complications in the elective admission group v. the emergency admission group included failed extubation in $5.7 \%$ v. $13 \%(p=0.006)$, chest infection on admission in $20.6 \%$ v. $45.0 \%$ 
$(p<0.0001)$, bloodstream infection in $3.8 \%$ v. $17.4 \%(p<0.0001)$, acute kidney injury in $17.4 \%$ v. $32.9 \%$ ( $p=0.001$ ), liver impairment in $3.5 \%$ v. $19.3 \%$ ( $p<0.0001)$, neurological sequelae in $1.9 \%$ v. $14.9 \%$ $(p<0.0001)$ and PICU readmission in $10.3 \%$ v. $30.4 \%(p<0.0001)$. In both the elective and emergency admission groups $4.1 \%$ of suffered a cardiac arrest during PICU stay $(p<0.0001)$. PICU mortality for the elective v. emergency admission group was $1.6 \%$ v. $12.4 \%(p<0.0001)$ and standardised mortality 0.25 v. 0.80 .

Conclusions. Overall, 34.9\% of all PICU admissions during 2015 were PCCC admissions. Emergency PCCC admissions have a higher morbidity and mortality in our setting.

\section{ALCAPA in children: Single-centre perioperative outcome}

\author{
B Rossouw, ${ }^{\star}$ M Wege, A C Argent, B Morrow, J Lawrenson \\ Division of Critical Care and Children's Heart Diseases, Red Cross War \\ Memorial Children's Hospital, University of Cape Town, South Africa \\ *beyra.rossouw@uct.ac.za
}

Background. A 1970s report on anomalous left coronary artery to pulmonary artery (ALCAPA) patients from our institution documented $50 \%$ hospital mortality. Current outcomes are not clear.

Objectives. To document perioperative management and outcome of children with ALCAPA, admitted to Red Cross War Memorial Children's Hospital PICU. No mechanical cardiac support was available.

Methods. A retrospective 10-year longitudinal study between July 2004 and August 2015 was conducted.

Results. A total of 46 patients were included, of which $45 \%$ presented in cardiogenic shock requiring immediate PICU admission. Forty-three underwent coronary artery reimplantation surgery. Median length of PICU stay, postoperative inotropic support and ventilation were 9.9, 8.3 , and 7 days, respectively. Perioperative morbidity included community-acquired infection (27\%), nosocomial infection (40\%), arrhythmias (24\%), cardiac arrest (13\%) and acute kidney injury needing dialysis $(6 \%)$. PICU mortality was $24 \%(n=7)$. A total of 39 (85\%) patients survived to hospital discharge, with 5 deaths after discharge. No independent predictors of mortality could be identified on multivariate analysis. Fourteen $(30 \%)$ patients needed PICU readmission/s and six (13\%) required cardiac reintervention surgery. For survivors, the median time to ejection fraction recovery was 15.5 months.

Conclusions. Coronary reimplantation is a successful procedure in our institution. Perioperative infection remains a major morbidity. Mechanical cardiac support could alter these findings.

\section{The impact of echocardiography in a paediatric intensive care unit (PICU)}

\section{B Rossouw, ${ }^{\star}$ M Wege, A C Argent, B Morrow, J Lawrenson Division of Critical Care and Children's Heart Diseases, Red Cross War Memorial Children's Hospital, University of Cape Town, South Africa *beyra.rossouw@uct.ac.za}

Background. Echocardiography has become an important tool to assess critical ill adults. The influence of echocardiography on the management of critical ill children is not well described.

Objectives. To review the impact of echocardiography in a singlecentre tertiary PICU.
Methods. A prospective review of all echocardiographic studies was done in a combined 22-bed medical and surgical tertiary PICU between February 2015 and January 2016.

Results. During this 1-year study period $15 \%$ of the total PICU population received an echocardiographic assessment during PICU stay. A total of 379 echocardiograms (ECGs) were done on 211 PICU patients, of whom 88 were known to have a cardiac condition prior to PICU admission. Sixty-three per cent $(n=238)$ of the ECGs were done by the cardiology department and $37 \%(n=141)$ by ICU staff formally trained in echocardiography. Indications for ECGs were: assessment of cardiac function, anatomical anomalies, pulmonary pressure, infective endocarditis and pericardial effusion in 146,101, 54, 19 and 5 studies, respectively. Postcardiac surgery ECGs were performed to assess cardiac function, residual lesions and pulmonary pressure in 118, 137 and 21 studies, respectively. New congenital heart lesions were diagnosed in 47 PICU patients for the first time in PICU. The newly diagnosed congenital heart lesions included right ventricular outflow tract obstructions in 12 patients, 6 left ventricular outflow tract obstructions and shunt lesions in 27 patients. Newly acquired heart lesions were diagnosed in 10 patients, in whom 5 were dilated cardiomyopathy and 5 infective endocarditis. Normal hearts were confirmed in $14 \%$, and $26 \%$ of the ECGs confirmed new pathology that did not require PICU treatment change. Overall, 39\% of the ECGs identified new anatomical or functional pathology that subsequently altered PICU management. Treatment modification included surgical intervention $(n=41)$, change in inotropes $(n=47)$, pulmonary hypertension treatment $(n=21)$, anti-failure treatment $(n=22)$, fluid management $(n=13)$, prostaglandin $(n=6)$ and infective endocarditis treatment change $(n=5)$. Four patients were found to have inoperable cardiac lesions and were palliated.

Conclusions. Echocardiography is a valuable diagnostic tool in PICU and often contributes to treatment modification.

\section{Children with fulminant dilated cardiomyopathy or myocarditis}

\section{B Rossouw, ${ }^{*}$ A C Argent, B Morrow, J Lawrenson}

Division of Critical Care and Children's Heart Diseases, Red Cross War Memorial Children's Hospital, University of Cape Town, South Africa

*beyra.rossouw@uct.ac.za

Background. Mechanical cardiac support and transplantation have improved long-term outcome of cardiomyopathy and myocarditis in children internationally.

Objectives. To review the outcome of children with fulminant dilated cardiomyopathy (DCMO) or myocarditis in a setting without readily available mechanical cardiac support and transplantation.

Methods. A retrospective 5-year review of consecutive PICU admissions of children with fulminant DCMO and myocarditis to Red Cross War Memorial Children's Hospital PICU between January 2010 and July 2015 was conducted.

Results. A total of 95 children with a median age of 27.8 months were included. All presented in Ross stage 4 cardiac failure (77\% in cardiogenic shock). Admission median lactate was $6.5 \mathrm{mmol} / \mathrm{L}$. Admission left ventricular ejection fraction was $<30 \%$ in $79 \%$ of patients, and 9 developed intracardiac clots. Aetiology was presumed viral myocarditis in $87 \%$ and idiopathic DCMO in $13 \%$. Adenovirus polymerase chain reaction (PCR) was positive in 28, parvovirus in 19 and multiple positive viral studies in 32 . The median number of ICU 
admissions per patient was 1.5 (range 1 - 5) and length of ICU stay was 14.9 days $(1-69)$. Overall, $55 \%$ required ventilation for a median of 8.1 days; $100 \%$ required inotropic support for a median of 8.2 days; $82 \%$ received milrinone, $78 \%$ dobutamine and $33 \%$ adrenaline infusions. The median maximum inotrope score was 21.9. Complications during ICU stay included acute kidney injury in $68 \%$, of which two patients needed dialysis, liver derangement in $43 \%$, neurological events in $25 \%$ and $34 \%$ suffered a cardiac arrest episode. Thirty-three per cent had arrhythmias, of which $27 \%$ needed electrical cardioversion and $57 \%$ needed drug treatment. A total of $63(66 \%)$ children survived to ICU discharge. The overall hospital survival was $47 \%$. Of the ICU survivors, the median number of ward readmissions was 3.7 (range 1 - 19). Total median length of ward stay was 23.2 days $(1-138)$.

Conclusions. In our setting without transplantation availability, DCMO and myocarditis are associated with significant duration of hospital stay, morbidity and mortality.

\section{Factors associated with survival to discharge of newborns in a tertiary hospital}

\section{E Ballot, ${ }^{*}$ T Chirwa}

University of the Witwatersrand, Johannesburg, South Africa

*daynia.ballot@wits.ac.za

Background. Neonatal deaths contribute significantly to mortality in children under 5 years of age. Measures to reduce neonatal mortality have become a health priority in South Africa. Information obtained through clinical and mortality audit is an essential part of quality improvement in healthcare, in order to develop targeted interventions to improve outcome.

Objective. To determine predictors of short-term survival in neonates. Methods. This was a retrospective review of an existing database. Binary logistic regression considering the outcome survived/died was done to determine predictors of survival.

Results. A total 5018 neonates $>400$ g admitted to a tertiary hospital (Johannesburg, South Africa) between 1 January 2013 and 31 December 2015 were analysed. Mean birth weight was $2148 \mathrm{~g}$ (standard deviation (SD) 972) and mean gestational age was 34.2 (4.8) weeks. Overall survival was $85.6 \%$ (4 294/5 018). The most common causes of death were prematurity (46.2\%), hypoxia (19.5\%) and infection (17.2\%). The strongest predictors of outcome were birth weight (odds ration (OR) 1.0, $95 \%$ confidence interval (CI) 1.0 - 1.01) and gestational age (OR 1.1, 95\% CI 1.05 - 1.17). Other predictors of survival included metabolic acidosis (OR 0.14, 95\% CI 0.09 - 0.20), hyperglycaemia (OR 0.31, 95\% CI 0.23 0.41 ), mechanical ventilation (OR $0.35,95 \%$ CI 0.28 - 0.46), major birth defect (OR 0.12, 95\% CI $0.08-0.18$ ), resuscitation at birth (OR 0.39, $95 \%$ CI 0.31 - 0.49) and caesarean section (OR 1.8, 95\% CI $1.44-2.25$ ). Conclusion. Measures to improve neonatal outcome should focus on delivery room management of neonates, care of preterm neonates and infection control.

\section{The role of the intensive care unit (ICU) nurse in antimicrobial stewardship in a private hospital}

\author{
I Rout, ${ }^{\star}$ P Brysiewicz \\ School of Nursing and Public Health, College of Health Sciences, \\ University of KwaZulu-Natal, Durban, South Africa \\ *joanrout@worldonline.co.za
}

Background. The care of the critically ill patient has become progressively challenging across the world, with increasingly resistant pathogens resulting in difficult-to-treat infections. This is compounded by the decreasing effectiveness of many antibiotics. Severe infection increases the length of time spent in an ICU, increases morbidity and mortality and increases healthcare costs. Antimicrobial stewardship has the aims of slowing resistance and protecting patients and the wider community through the promotion of correct antimicrobial use by education and guidelines. Infection control measures are an essential part of antimicrobial stewardship in preventing emergent resistant pathogens and hospital-acquired infections. This has become a large part of nursing responsibilities in the ICU but the nurse does not yet have clear responsibilities with regards to antimicrobial therapy and the role that the nurse should play in antimicrobial stewardship.

Objectives. To identify of the role of the ICU nurse in an antimicrobial stewardship team.

Methods. A qualitative approach was used to obtain meaningful contributions that a questionnaire may not have been able to provide. Purposeful sampling was used to identify participants in a general ICU in the private healthcare sector. Semistructured interviews were conducted with 15 participants, namely ICU nurses, nursing management, surgeons, anaesthetists, physicians, microbiologists and pharmacists. Data were analysed and categorised using content analysis. Trustworthiness was demonstrated by meeting the required qualitative criteria of credibility, transferability, dependability and confirmability. Results. Perspectives of the members of the multidisciplinary antimicrobial stewardship team identified the role of the ICU nurse in an antimicrobial stewardship team as being organisational, advocatory, clinical and collaborative. Suggestions were made to further develop this nursing role by supporting proactive behaviour, teaching and learning, and teamwork. Concerns were raised about this role relating to resource barriers, knowledge deficit, poor attitude towards work, ineffective teamwork, working in isolation and economic pressures.

Conclusion. The ICU nurses' role in antimicrobial stewardship should be recognised, developed and supported by all healthcare stakeholders as it is essential for the successful implementation of an antimicrobial stewardship programme within this acute healthcare setting. The factors affecting the development of this role need to be further explored.

\section{Elevated lactate from three different mechanisms - a case series}

\author{
$\underline{\text { S Omar }},{ }^{\star}$ K Naidoo, R Gheevarghese \\ Intensive Care Unit, Baragwanath Academic Hospital and University of \\ the Witwatersrand, South Africa \\ *shahedicu@gmail.com
}

Background. Traditionally considered due to tissue hypoxia, lactate is an important marker for shock and poor prognosis in critically ill patients. Biochemically, lactate production is associated with proton generation. Using a quantitative physicochemical approach, others have argued that unmeasured anions rather than lactate account for the majority of the metabolic acidosis in patients with elevated lactate.

Objective. To characterise the different components of the metabolic acidosis as lactate increases and/ or decreases from three different causes of elevated lactate-associated metabolic acidosis.

Methods. A case series with three patients found to have metabolic acidosis and increased blood lactate concentration was studied. The first case was from cardiogenic shock, the second from metformin 
toxicity and the third from septic shock. We evaluated simultaneous blood gas, electrolytes, calcium, magnesium, phosphate, creatinine and lactate concentrations over the 24-hour period from onset to resolution of the problem. Using a quantitative physicochemical approach described previously, we calculated apparent strong ion difference (SIDa), effective strong ion difference (SIDe) and the strong ion gap (SIG). Next we calculated the total standard base deficit (SBD) acid and its individual components, namely SBD chloride, SBD $\mathrm{H}_{2} \mathrm{O}, \mathrm{SBD}$ lactate and SBD SIG. Results. The three patients all developed significant metabolic acidosis with a median $\mathrm{pH}$ of 7.17 and SBD of $20.1 \mathrm{meq} / \mathrm{L}$. At a pH below 7.25 there was a significant increase in the total SBD acid $(p=0.000)$. SBD chloride and SBD lactate increased significantly $(p<0.05)$. There was a nonsignificant decrease in SBD SIG and SBD $\mathrm{H}_{2} \mathrm{O}$. Unmeasured anions, estimated by SBD SIG, contributed a maximum of $9.75 \%$ to the total SBD acid and were reduced to zero at the peak lactate concentration, which coincided with minimum $\mathrm{pH}$ and maximum SBD in all three cases. Although the absolute SBD chloride concentration increased as the acidosis worsened, the proportion $/ \%$ of SBD chloride was inversely related to the proportion/\% of SBDlactate (Spearman's rho $=0.92, p<0.05$ ). Conclusion. Lactate, not unmeasured anions, was the major contributor to the metabolic acidosis, irrespective of the mechanism of elevation. Chloride's contribution to the acidosis also increased, except for the patient undergoing renal replacement therapy.

\section{Development of a paediatric simulation programme}

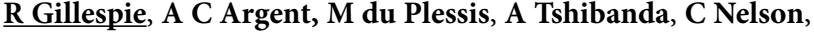 M McCulloch* \\ Red Cross War Memorial Children's Hospital, University of Cape Town, South Africa \\ *mignon.mcculloch@uct.ac.za}

Background. Simulation (Sim) is a form of interactive training which can be used for technical skills teaching, human factors interaction, and improving clinical governance and patient safety.
Objective. To describe a new paediatric Sim programme, which commenced in February 2014 and is now a regular event in our hospital training programme.

Methods. Initial training of instructors was done by an international team during a 1-week interactive session in February 2014 (supported by funding from the Critical Care Society of Southern Africa). Local faculty (including doctors, nurses and clinical technologists) was developed with no additional resources. Intermediate fidelity equipment was available initially, while high-fidelity equipment was donated. The concept was presented at a 'grand rounds' to get 'buy-in' from hospital and administrators before introducing simulations into the hospital environment.

Results. Over 2 years, simulation provided training in three areas: (i) technical skills and human factors in the paediatric intensive care unit (including nitric oxide use, ventilation and continuous veno-venous haemodialysis); (ii) 'Sim on request' from the rest of the hospital including resuscitation training, emergency case management in emergency departments and 'how to manage angry parents'; and (iii) anaesthetist-run Sims in theatre performing specific orchestrated case scenarios on a regular basis. Simulation was used to develop plans and prepare an oncology ward for the introduction of desensitisation therapy for asparaginase allergy. It was used by a nurse-led team to develop processes for 'turning the complex patient while connected to monitors'. There has been an increased demand for simulation as a development and training tool. Our experience suggests that this is a powerful tool to develop appropriate processes at clinical level, to train new personnel, and to help personnel (and managers) understand the dynamics of clinical care.

Conclusion. After initial investment in staff training and equipment, it has been possible to establish a paediatric Sim programme that appears to be an effective tool for addressing a range of issues in clinical practice, including: protocol development, staff training (in both technical and non-technical skills) and staff debriefing. Further analysis is needed to assess sustainability of this programme and the results thereof. 